\title{
Teoría aristotélica de la responsabilidad moral
}

Daria Peña Rávago

Pontificia Universidad Católica del Perú

Resumen: El presente trabajo tiene como propósito elucidar la postura de Aristóteles con respecto a la responsabilidad moral basándonos en el contenido de su libro Ética a Nicómaco. A pesar de que no encontramos una teoría explícita al respecto, demostraremos que Aristóteles habla de lo que hoy en día llamaríamos responsabilidad moral en términos de voluntariedad y capacidad de deliberación. Por ello, examinaremos en primer lugar su teoría de la voluntariedad; luego, analizaremos el papel que le otorga al deseo, la deliberación y la elección en la responsabilidad y, finalmente, profundizaremos en su análisis de la naturaleza del carácter del agente.

Palabras clave: responsabilidad, ética, voluntariedad, deliberación, carácter

Abstract: "Aristotle's Theory of Moral Responsibility". The purpose of this paper is to elucidate Aristotle's position on moral responsibility on the basis of the contents of his work Nicomachean Ethics. Although there is no explicit theory on the subject, here we show that Aristotle speaks in terms of voluntariness and capacity for deliberation what we nowadays call moral responsibility. Therefore, we first examine his theory of voluntariness; then, we analyze the role he gives to desire, deliberation and choice in responsibility and, finally, we deepen his analysis of the nature of the agent's character.

Keywords: responsibility, ethics, voluntariness, deliberation, character 
A lo largo de Ética a Nicómaco se puede observar el profundo interés de Aristóteles por aclarar no solo la naturaleza de las virtudes y los vicios, sino también las condiciones bajo las cuales uno merece ser objeto de alabanzas o reproches debido a sus acciones, ya sean virtuosas o viciosas. Por lo tanto, es muy probable que haya escrito este texto con el objetivo de optimizar el sistema de justicia de su época, promoviendo que la ley sea la encarnación de las virtudes. En efecto, hace alusiones a la ley ateniense cuando vincula su teoría de la acción al ámbito legal que se encarga de honrar y castigar dependiendo si la persona actúa o no de acuerdo a lo que dicta la recta razón (Aristóteles 1985, 1109b30-35). Así pues, si bien Aristóteles no elaboró explícitamente una teoría de la responsabilidad moral, sí investigó acerca de la agencia humana y de la manera en la que cada uno debe ser juzgado por sus acciones. Por ello, en el presente trabajo analizaremos la postura de Aristóteles con respecto a la responsabilidad moral demostrando que él la mide en términos de voluntariedad y capacidad de deliberación. Es a partir de estos dos criterios, el grado de voluntad y de elección del agente, que diferencia cuándo un acto debe ser objeto de alabanzas, reproches, indulgencia o compasión.

A su vez, esto implica que, desde su perspectiva, la acción no debe ser examinada de manera aislada, debido a que la elección y el agente son igualmente fundamentales. Es por esto que no vamos a encontrar en su pensamiento un conjunto de mandamientos o leyes fijas que nos digan qué debemos y qué no debemos hacer, como tampoco una teoría universal de la moralidad que se pueda determinar a priori. La ética tiene como objeto las cosas particulares y contingentes, aquellas que son pero pueden ser de otra manera. Por estos motivos, en un mundo tan complejo, ambiguo y abierto a las posibilidades como el nuestro, lo que busca Aristóteles no es el bien absoluto, sino una filosofía práctica que nos guíe hacia la formación del hábito del buen vivir. Él desarrolla una ética de las virtudes, de modo que una praxis moral nos ayude a encontrar la felicidad.

Sin embargo, esto es mucho más complejo de lo que puede parecer a primera vista, por ejemplo, una acción puede ser voluntaria pero no elegida. Por esto, sostenemos que existen distintos grados de responsabilidad que se explican a lo largo del libro y podemos tomar como punto de referencia. 
Con el fin de resolver el problema, debemos preguntarnos cuáles eran las motivaciones del agente a la hora de actuar, qué intenciones eligió, si deliberó correctamente o no, cuáles fueron sus reacciones emocionales, si actuó de acuerdo a la virtud por sí misma o por su propio interés, etc. Asimismo, como resalta Nussbaum, "(f)uera del contexto inmediato, necesitamos preguntar cómo la elección encaja en patrones de elección y respuesta que esta persona ha cultivado (o no)" $(1999,582)$. Esto último es esencial para comprender la psicología de los agentes y si la acción juzgada encaja en su comportamiento habitual. Para responder a estas preguntas y argumentar hasta qué punto podemos considerar Ética a Nicómaco una teoría de la responsabilidad moral tomaremos en cuenta sus tres pilares: en primer lugar, examinaremos la teoría de la voluntariedad de Aristóteles; luego, analizaremos el papel que le otorga al deseo, la deliberación y la elección en la responsabilidad; $y$, finalmente, profundizaremos en su estudio acerca de la naturaleza del carácter del agente.

\section{§I. La explicación de la acción}

En primer lugar, nos dedicaremos a los distintos tipos de acciones, distinguiendo entre las acciones voluntarias (hekoúsios), involuntarias (akoúsios) y no voluntarias. En Ética a Nicómaco, Aristóteles realiza una definición negativa de la voluntad, es decir, comienza explicándola a partir de aquello que no es.

Así pues, dentro de las acciones involuntarias se encuentran aquellas realizadas por fuerza o por ignorancia. En el primer caso, describe lo forzoso como "aquello cuyo principio es externo, sin que el hombre forzado intervenga en nada" (Aristóteles 1985, 1110b16-17). Aquí Aristóteles hace referencia al principio del movimiento o arché: la causa eficiente que da origen a la conducta. Si el arché es externo podemos decir que la acción fue hecha por fuerza, como por ejemplo cuando un hombre que tiene poder sobre nosotros nos domina. No obstante, cabe aclarar que esto no aplica en el caso del actuar por cosas agradables y hermosas. A pesar de que efectivamente intervienen factores externos en ambas situaciones, Aristóteles hace énfasis en que en la segunda no podemos culpar a las 
causas externas porque la fuerza que nos lleva a actuar se encuentra de todos modos en nosotros mismos: actuamos por nuestro placer. Entonces, podemos deducir que si actuar por placer sí es voluntario, el agente en estos casos es, por lo tanto, responsable de sus acciones. Actuar por la fuerza en sentido involuntario implica, en cambio, actuar en contra de la propia voluntad y dolorosamente. Por lo tanto, en estas circunstancias el sujeto no sería responsable en sentido moral.

El segundo caso de involuntariedad son las acciones que se llevan a cabo por ignorancia, y Aristóteles las divide a su vez en dos clases:

Todo lo que se hace por ignorancia es no voluntario, pero, si causa dolor y pesar, es involuntario. En efecto, el que por ignorancia hace algo, cualquier cosa que ello sea, sin sentir el menor desagrado por su acción, no ha obrado voluntariamente, puesto que no sabía lo que hacía, pero tampoco involuntariamente, ya que no sentía pesar. Así, de los que obran por ignorancia, el que siente pesar parece que obra involuntariamente, pero el que no lo siente, ya que es distinto, <digamos que ha realizado un acto> al que llamaremos «no voluntario» (Aristóteles 1985, 1110b18-24).

Como podemos observar, Aristóteles diferencia las acciones involuntarias de las no voluntarias basándose en la respuesta emocional del agente ante lo que hizo. ¿Cuáles son las consecuencias de esta división? A partir de esto podemos suponer que respecto a la responsabilidad moral el filósofo sostendría que a la hora de juzgar a una persona no solo es importante tener en cuenta las acciones cometidas, sino también el conocimiento que tenía de las circunstancias y si aquellas le causan dolor o no. Entonces, si bien en ambos casos el origen de la acción está en el agente y este no sabía lo que hacía, las acciones involuntarias son aquellas en las que sí se muestra arrepentimiento y las no voluntarias, en las que no. Esto podría significar que las acciones no voluntarias cargan con una responsabilidad moral mayor porque no demuestran síntomas de remordimiento cuando se conocen las circunstancias particulares que antes no se sabían. Las emociones del agente cumplen un rol fundamental, por lo que no debemos juzgar de la misma manera a aquellos con una reacción de pesar ante sus actos y a quienes son indiferentes o incluso se alegran. Como dice Aristóteles: "no por ello los autores son injustos ni malos, porque el daño no tiene por causa la maldad; pero cuando actúan con intención, son injustos y malos" (1985, 1135b25). 
En efecto, es probable que en los casos de actos involuntarios por ignorancia la persona hubiese obrado de otra manera si hubiera podido, lo que la libera de una parte de la carga moral.

Además, podemos encontrar en la involuntariedad una tercera distinción: la ignorancia de lo universal y de lo particular. Aristóteles vincula la primera con la maldad porque es para él un estado de ignorancia universal en el cual el sujeto no sabe lo que debe y no debe hacer. No obstante, no hay que entender su ética como el cumplimiento de acciones morales por deber, sino como la formación de hábitos virtuosos para vivir una vida buena. Aristóteles compara la maldad a la embriaguez y a la cólera (1985, 1110b25-27). De hecho, uno actúa en tales casos no por ignorancia, sino con ignorancia y por los efectos del alcohol y de la cólera, respectivamente. En ambos casos se actúa por una fuerza externa, pero esto no puede eximirnos de la responsabilidad porque se ha elegido permanecer en la ignorancia. La causa de la acción es el vicio, no la ignorancia, y el vicio se adquiere practicándolo. Tanto emborracharse como dejarse llevar por la cólera son vicios que pudieron educarse a través del control de las pasiones. Es por esto que la maldad puede ser, como sostiene Baracchi, el resultado de una mala crianza y de otras circunstancias desfavorables $(2008,139)$. No obstante, uno no puede utilizar esto como justificación para librarse de la responsabilidad de sus actos porque una persona adulta tiene alternativas para reformar su carácter, como veremos más adelante.

Con respecto a la ignorancia de lo particular, esta se refiere al desconocimiento de las circunstancias en las que se realiza la acción: el sujeto, el objeto, el instrumento, el lugar, el tiempo, la manera y la causa. Este tipo de ignorancia es la propiamente involuntaria para Aristóteles y debe ir seguida de pesar y arrepentimiento. Entonces, si por ejemplo uno dice algo sin saber que estaba revelando un secreto (Aristóteles 1985, 1111a9), lo hace involuntariamente porque ignoraba las circunstancias. Si bien Aristóteles no lo explicita, podemos inferir que las personas que llevan a cabo una acción por ignorancia particular de la situación y se arrepienten tienen un grado de responsabilidad moral menor, ya que la ignorancia es la causa de la acción. Así pues, como concluye Bravo, "la ignorancia excusa la voluntariedad si y solo si: (1) es ignorancia de lo particular; (2) es causa de la acción, 


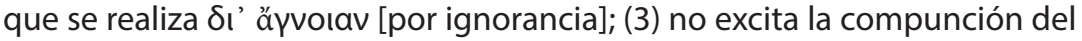
agente" $(2004,8)$.

Existen también acciones mixtas, en las que no está claro si son voluntarias o involuntarias, como cuando se actúa de un modo infame por temor a males mayores o por alguna causa noble. Si bien en un sentido absoluto parecerían involuntarias porque nadie las elegiría por sí mismas debido a que causan dolor y pesar, en estos casos las acciones son voluntarias porque el principio del movimiento (arché) se encuentra en el sujeto y es él el que decide llevarlas a cabo o no (Aristóteles 1985, 1110a15-16). Sin embargo, si bien se afirma que se parecen más a las voluntarias, no cae necesariamente toda la carga moral sobre el agente. Aristóteles sostiene que cuando se soporta algo vergonzoso o doloroso por causas grandes y nobles, las personas que las realizan son alabadas, y cuando se trata de acciones que no se deben hacer, pero aun así se realizan debido a causas que escapan de su control, las personas son perdonadas. En consecuencia, pareciera que Aristóteles le resta responsabilidad al sujeto a pesar de que el arché de la acción se encuentra en él mismo y de que al fin y al cabo lo eligió. Probablemente esto se debe a que el filósofo comprende que en determinadas circunstancias uno no tiene la absoluta capacidad de elegir, ya que está presionado por una serie de elementos externos que dependen del momento preciso (kairós). No obstante, se nos presenta una tercera evaluación de las acciones mixtas, aquellas a las que según Aristóteles uno no puede ser forzado y no obtienen nunca un perdón. A este grupo pertenecen acciones como matar a la madre. Evidentemente, no hay nada que pueda justificar algo así ni ninguna fuerza lo suficientemente fuerte como para obligarnos. En consecuencia, si alguien realiza una acción de este tipo, sí recaería en él la responsabilidad moral, porque sin importar las circunstancias siempre será preferible elegir la propia muerte. A partir de esto podemos deducir que para Aristóteles no hay una manera absoluta de determinar la responsabilidad moral, sino que es necesario analizar las circunstancias particulares.

Finalmente, para Aristóteles son voluntarias aquellas acciones que (a) tienen su principio en el agente mismo y (b) en las que él es consciente de las circunstancias concretas en las que se produce la acción (1985, 1111a22-24). 
Pero esta postura ha sido criticada, bajo el argumento de que el hecho de que el origen de la acción sea interno no es suficiente para que algo dependa de uno ni para que sea voluntario, apoyándose en la excepción que nota Aristóteles en los procesos naturales como sudar, crecer o digerir (Irwin 1980, 122). Si bien estas acciones tienen el origen en uno mismo, no son voluntarias porque no depende de nosotros llevarlas a cabo o no. Asimismo, Irwin reprueba también que Aristóteles haya omitido el papel del deseo en las acciones voluntarias en Ética a Nicómaco, asumiendo que lo daba por sentado. Sin embargo, a nuestro parecer, Aristóteles sí toma en cuenta el papel del deseo del agente. De hecho, señala que para poder juzgarlo es importante considerar sus reacciones emotivas al conocer las consecuencias de un acto que realizó con ignorancia. Por este motivo, Aristóteles considera fundamental la presencia de arrepentimiento luego de una acción realizada por ignorancia para que esta sea considerada involuntaria y el agente sea perdonado. En todo caso, una versión revisada de la definición de Aristóteles que Irwin llama la teoría "simple" sería así: A hace x voluntariamente si y solo si (a) A cree que x es $\mathrm{F}$; (b) x es $\mathrm{F}$; (c) las creencias y deseos de A, como razones, llevan a A a hacer x (Irwin 1980, 124).

No obstante, señala que todavía se pueden encontrar incongruencias en este modelo: $x$ puede ser $\mathrm{F}$ y también $\mathrm{G}$, de manera que $\mathrm{A}$ puede hacer $\mathrm{x}$ tanto voluntaria como involuntariamente si desconoce que $x$ es también G. Por ejemplo, A le prepara un queque de maní a B y cuando B lo come se asfixia porque tiene alergia al maní. La acción de $A$ es voluntaria en tanto le entregó el queque a $B$ para que lo comiera, pero también es involuntaria porque al no saber de la alergia de B no sabía que se asfixiaría. En consecuencia, a pesar de la aparente contradicción, A causó la asfixia de $B$ involuntariamente. Por ello, si bien A es responsable en sentido causal, no es moralmente responsable porque no tenía la intención de hacerlo ni sabía que algo malo pasaría.

En suma, esta distinción entre actos voluntarios e involuntarios es fundamental para esclarecer la teoría de la responsabilidad moral porque para Aristóteles un acto ético es siempre voluntario. En efecto, para que haya un juicio ético uno debe ser consciente de lo que está escogiendo. En cambio, "cuando se hacen involuntariamente no se actúa ni justa ni injustamente 
excepto por accidente, pues entonces se hace algo que resulta accidentalmente justo o injusto" (Aristóteles 1985, 1135a16-19). Por estos motivos, las acciones voluntarias son alabadas o reprochadas, mientras que las involuntarias son, al contrario, objeto de indulgencia o compasión. Cuando el acto es involuntario, si bien la cosa es injusta, esta no llega a ser una acción injusta y, por lo tanto, evaluaremos si hay responsabilidad o no dependiendo de las circunstancias. Como hemos visto a lo largo de esta sección, debido a la multiplicidad de matices dentro de las acciones voluntarias, no podemos trazar una línea perfecta entre lo voluntario y lo involuntario. Por ello, esto nos lleva a entender la voluntariedad más bien como un continuum. Sin embargo, aún nos queda una duda. Si las acciones voluntarias son aquellas que no se realizan por una fuerza externa, ¿qué es lo que mueve al sujeto a actuar? Además, ¿si admitimos que los niños y animales actúan voluntariamente, significa que son también responsables de sus acciones? A continuación, responderemos a esta pregunta mediante un análisis del papel que le otorga Aristóteles al deseo y a la razón en las acciones morales.

\section{§II. El papel de la elección en la responsabilidad}

La elección (proaíresis) es la segunda condición para que un sujeto pueda ser considerado responsable moralmente de sus acciones. Si bien podría ser confundida con lo voluntario, cabe resaltar que no son lo mismo: es cierto que toda elección es voluntaria; sin embargo, no todo lo voluntario se elige. Así pues, "de lo voluntario participan también los niños y los otros animales, pero no de la elección, y a las acciones hechas impulsivamente las Ilamamos voluntarias, pero no elegidas" (Aristóteles 1985, 1111b7-8). En efecto, la elección no abarca toda la dimensión de lo voluntario, sino solo los juicios a los que se llegan después de la reflexión acerca de lo que es mejor para el panorama de nuestros fines en conjunto: es una subclase de las acciones voluntarias. Pero no es un proceso puramente racional. Se elige efectivamente lo que se ha decidido después de una deliberación, pero son los deseos los que por lo general determinan nuestros fines. Por lo tanto, la elección puede definirse como "un deseo deliberado de cosas 
a nuestro alcance" (Aristóteles 1985, 1113a11) a partir del cual decidimos cómo actuar.

Al igual que con lo voluntario, Aristóteles empieza su descripción de la elección especificando aquello que no es, a partir de una crítica a las teorías de sus predecesores sobre la naturaleza de la misma. La elección no es un apetito (epithunía) ni un impulso (thumóv), porque estas conductas son propias más bien de los irracionales y los acráticos, quienes no actúan eligiendo, sino arrastrados por sus pasiones. Tampoco es equivalente a una opinión (doxa). La opinión puede referirse a cualquier cosa, ya sea posible, imposible, externa a nosotros o que esté a nuestro alcance, y se distingue también por ser verdadera o falsa. En contraste, la elección es buena o mala y elegimos sobre lo que estamos seguros, guiados por la razón y la reflexión, no como sucede con la opinión, ya que a veces opinamos sobre cosas que no conocemos totalmente. Del mismo modo, a diferencia de las elecciones, las opiniones no determinan nuestro carácter, porque a pesar de tener opiniones verdaderas, una persona puede no elegir lo que debe debido a algún vicio.

La elección no es, pues, nada de esto. Sus dos componentes principales son el deseo (órexis) y la deliberación (boúleusis). Se cree que Aristóteles inventó el término órexis para introducir una característica común a todos los movimientos animales dirigidos a un objeto. Es una noción general de "querer" que se opone a una concepción pasiva del deseo connotando, en cambio, actividad: "es un ir a por algo, perseguirlo (física o mentalmente)" (Nussbaum 1995, 355). El deseo pertenece a la parte irracional del alma y la deliberación a la racional, pero no están completamente incomunicados. Mientras que el deseo se refiere al fin (telos), la elección se enfoca sobre todo en los medios que conducen al fin (pros to telos). En efecto, podemos desear ser felices pero no elegir serlo, debemos en cambio elegir los medios que nos ayudarán a alcanzar la felicidad. Asimismo, la elección se hace solo de aquello que es posible y realizable por uno mismo. De esto se sigue que uno pueda desear lo imposible, como por ejemplo ser inmortal, pero no escoger algo así. Para que la elección no siga los deseos descontroladamente, es fundamental tener en cuenta el papel del razonamiento. El deseo participa de la racionalidad en tanto se dice que puede escuchar al logos, 
lo que da lugar a un deseo inteligente o procesado por una deliberación (bouleutiké órexis). En palabras de Aristóteles, "cuando decidimos después de deliberar, deseamos de acuerdo con la deliberación" (1985, 1113a11). Como plantea Nussbaum, la boúlesis es un tipo de órexis que se origina en la parte racional del alma, un querer que se produce luego de la deliberación (1995, 356). A diferencia de los animales, los humanos tenemos la capacidad de orientar nuestros deseos al bien, en otras palabras, podemos aplicar el logos a nuestras pasiones.

En cuanto a la deliberación, esta consiste en un cálculo racional acerca de lo que es mejor para uno de acuerdo a las prioridades que uno tenga y a los fines que busque alcanzar, el cual necesita saber cómo, por qué y qué tenemos que elegir. En otras palabras, es la razón direccionada en vista de algo. Al ser movida por el deseo, se encarga de deliberar acerca de los mejores medios para alcanzar los fines. Este proceso es llamado silogismo práctico, el cual consiste en dos premisas que representan al deseo y al intelecto y a las cuales les sigue una acción. En cuanto al objeto de deliberación, Aristóteles sostiene que deliberamos "sobre lo que está en nuestro poder y es realizable" $(1985,1112$ a 32-33) y "acerca de cosas que suceden la mayoría de las veces de cierta manera, pero cuyo desenlace no es claro y de aquellas en que es indeterminado" (1985, 1112b 8-10). Efectivamente, sería inútil deliberar acerca de las cosas sobre las cuales no tenemos poder alguno, sobre conocimientos exactos o sobre cosas que no van a cambiar. Asimismo, como ya habíamos señalado anteriormente, en principio "el deseo se refiere más bien al fin, la elección a los medios conducentes al fin" (Aristóteles 1985, 1111b27). Sin embargo, ¿es cierto que no deliberamos acerca de los fines? ¿Acaso no reflexionamos acerca de lo que es más importante lograr para nosotros?

Efectivamente, esta traducción ha sido cuestionada y se ha propuesto otra que da lugar a una concepción más amplia de la razón, según la cual deliberamos "sobre las cuestiones concernientes a los fines". Cuando Aristóteles escribe "ni el médico delibera sobre si curará (...) sino que, puesto el fin, consideran cómo y por qué medios pueden alcanzarlo" (1985, 1112b14-15; cursivas añadidas), nos lleva a pensar que el fin ya ha sido establecido y una vez tomada la decisión, esta no será cuestionada. Tal y como Wiggins resalta, 
sería absurdo suponer que una persona no pudiera deliberar acerca de qué profesión escoger y tampoco es probable que Aristóteles haya creído esto $(1980,226)$. En el ejemplo del médico, él evidentemente no delibera acerca de si curará porque ese fin ya lo escogió a la hora de decidir dedicarse a la medicina. En efecto, su fin general está implícito en su propia definición: profesional dedicado a la atención de la salud. Guariglia hace un paralelo en este punto con el ejercicio de la virtud, según el cual "el hombre justo no se cuestiona los fines que el ejercicio de la justicia como tal le impone, pues tal cosa carece de sentido, sino que se pregunta en las circunstancias $a, b, c$ qué transacción entre él y los otros agentes involucrados constituye un acto de justicia" (1997, 209). Por consiguiente, es una posibilidad que Aristóteles no haya querido decir que es imposible deliberar acerca de los fines, sino que toda deliberación tiene un propósito y en el momento de deliberar acerca de los medios para alcanzarlo ya no lo cuestionamos porque ya lo hemos establecido previamente. Los únicos fines que Aristóteles declara expresamente fuera de toda deliberación son la salud y la felicidad (1985, 1111 b26-29). Así pues, sí existe una deliberación acerca de los fines vinculada a nuestras prioridades. De hecho, debemos deliberar para decidir qué fines priorizar y cuáles subordinar a otros. Todos los fines están articulados entre sí en miras de un fin mayor.

Esto está relacionado a la responsabilidad moral en tanto que partiendo de esta interpretación, según la cual la elección también apunta al fin pero se encarga sobre todo de conseguir los medios para alcanzarlo, podemos decir que la elección representa la intención moral del agente. Entonces, la elección sería la causa eficiente de la acción y la causa formal de la responsabilidad (Bravo 2004, 13). Sin la elección no hay pues responsabilidad, por lo que una acción es responsable si y solo si es elegida. No obstante, en la sección anterior habíamos visto que uno puede ser responsable también de las acciones que realiza involuntariamente. ¿Qué sucede con la elección en estos casos? ¿Acaso ya no es un requisito para la responsabilidad? A pesar de que Aristóteles no responde estas preguntas, creemos que estaría de acuerdo con resolver esta aparente contradicción considerando que la responsabilidad aplica no solo a aquellos que actúan a partir de sus elecciones sino también a aquellos que tienen la capacidad de elegir. Así pues, si alguien no actuó según una deliberación, es a pesar de eso responsable 
porque pudo haberlo hecho. Como lo explica Esponda, esto "se debe a que sus acciones están ya referidas a un cierto modo de vida que ha sido deliberado y elegido con anterioridad" $(2016,143)$. En otras palabras, no estamos deliberando constantemente antes de realizar cualquier acción, pero aun así somos responsables de estas porque las hacemos como resultado de nuestro carácter. Teniendo en cuenta estos detalles, A es responsable por hacer $x$ si y solo si (a) A es capaz de decidir efectivamente acerca de $x, y$ (b) A hace $x$ voluntariamente (Irwin 1980, 132).

Esto es a lo que Irwin llama la "teoría compleja", en la que "corrige" la teoría simple de Aristóteles. Desde su punto de vista, la teoría simple es incompleta e induce a considerar responsables de sus acciones a los niños y animales. En nuestra opinión, el problema de esta interpretación recae en que Irwin considera la teoría de la voluntariedad de Aristóteles como equivalente a una teoría de la responsabilidad, cuando no es así. El estagirita manifiesta en múltiples ocasiones la importancia de complementar la voluntariedad con la deliberación y la elección, esto es finalmente lo que nos brinda la capacidad de actuar moralmente. Entonces, tanto los animales como los niños y los adultos que no tienen capacidad deliberativa serían responsables en sentido causal si sus acciones son voluntarias, pero no se los puede juzgar moralmente.

Igualmente, dado que las acciones responsables pueden ser juzgadas como virtuosas o viciosas, al hablar de elecciones estamos hablando también del carácter del agente. De hecho, de acuerdo con Aristóteles la virtud y el vicio son voluntarios. Así pues, juzgar las elecciones es para Aristóteles la mejor manera de conocer el carácter de una persona (1985, 1111b5-7), por lo que es importante considerar también el papel del carácter si queremos hablar de responsabilidad moral.

\section{§III. El carácter}

En efecto, las elecciones voluntarias elegidas revelan las disposiciones de carácter (héxis) viciosas o virtuosas a partir de las cuales la persona actúa. Por ello, debemos detenernos a examinar el carácter para encontrar los 
fines que persigue el agente y ver si estos coinciden con sus acciones. En palabras de Nussbaum, la filosofía moral debe "interesarse por la motivación y la intención, la emoción y el deseo: en general, por el carácter de la vida moral interior y por patrones establecidos de motivación, emoción y razonamiento que nos conducen a considerar a alguien una persona de cierto tipo (valiente, generosa, moderada, justa, etc.)" (1999, 578). Así pues, hay que mirar la vida ética del agente en su totalidad para poder captar sus patrones, intenciones y características. Sin embargo, a raíz de esta cuestión pueden surgir varias dudas. En primer lugar, si bien afirmamos que uno es responsable de sus acciones cuando actúa en base a sus creencias y deseos, ¿podemos elegir nuestras creencias y deseos? De no ser así, ¿somos responsables de ellos? También, ¿en qué medida somos responsables sobre nuestros modos de ser (héxis)? ¿Deberíamos exigirle lo mismo a todos si cada uno ha recibido una educación diferente? A lo largo de esta sección vamos a intentar resolver estas cuestiones.

Con respecto a las disposiciones de carácter, Aristóteles plantea que "las acciones [...] son las principales causas de la formación de los diversos modos de ser" $(1985,1103$ b30). Esto quiere decir que la costumbre es fundamental en la adquisición de la virtud: es así como se forman los hábitos, haciendo. No obstante, esto no elimina el rol de la naturaleza, ya que "las disposiciones se logran a partir del perfeccionamiento que se da en la ejecución de una capacidad que sí es natural" (Esponda 2004, 146). Así pues, hay personas que se inclinan más a ciertas virtudes que otras, por lo que debemos tener en cuenta la naturaleza de las pasiones del agente a la hora de juzgarlo.

De este punto se desprende el tema de la educación, la cual es entendida por Aristóteles como la formación de hábitos morales a partir del placer y del dolor. Debemos aprender a sentir dolor ante las cosas malas y placer ante las buenas. Pero esto no hay que entenderlo como un adoctrinamiento, porque cada uno actúa según su propia percepción del bien. De no ser así, entonces no podríamos decir que alguien es responsable moralmente nunca, pues el agente no sería dueño de su carácter. Si bien en un primer momento uno se habitúa como resultado de la repetición de ciertas acciones por orden de un tutor, con el paso del tiempo estas conductas logran afirmarse siempre y cuando el agente elija seguirlas por su propia razón 
o intención deliberada y no por imposición de otros, debe volverse una "segunda naturaleza". En cambio, si uno se abstiene de hacer algo malo por miedo al castigo, significa que no ha interiorizado la virtud. Como postula Burnyeat, uno no ha aprendido realmente una hábito por sí mismo hasta que no lo valore o incluso ame por sí mismo, lo que produce en consecuencia que le dé placer hacerlo (Burnyeat 1980, 78). Cabe resaltar que el fin de la acción no es el placer, sino que el placer acompaña al fin, lo potencia.

El problema reside en que no todos reciben una educación adecuada que les pueda brindar una idea correcta acerca de lo que es noble y justo. Aristóteles reconoce esta desigualdad de condiciones admitiendo que "es difícil encontrar desde joven la dirección recta hacia la virtud, si uno no se ha educado bajo tales leyes" (1985, 1179b32-33). No obstante, "que nuestro carácter esté influenciado por el tipo de educación que hemos recibido desde jóvenes y el modo en que nos hemos acostumbrado a llevar a cabo nuestras acciones, no significa que el carácter de un agente está necesariamente determinado por elementos externos" (Esponda 2004, 148). Sucede pues que algunos se encuentran en ventaja gracias a la buena fortuna, pero esto no es suficiente para determinar un buen carácter. El estagirita señala en efecto que "la situación ventajosa también se cree que contribuye a la magnanimidad", sin embargo, "también los que poseen tales bienes se vuelven altaneros e insolentes, porque sin virtud no es fácil llevar convenientemente los dones de la fortuna" (1985, 1124a21-31). Por lo tanto, podemos suponer que para Aristóteles uno debe ser considerado responsable moralmente de sus acciones independientemente de la educación que haya recibido, porque tanto los vicios como las virtudes son voluntarias. Dicho de otra manera, la responsabilidad individual posibilita que nuestro comportamiento no esté completamente determinado por factores naturales y las costumbres.

Aquí entra en el debate la cuestión de la akrasía. Los acráticos o incontinentes son aquellos que actúan en contra de su mejor juicio dominados por sus pasiones, como si estas cedieran no al argumento sino a la fuerza. Pareciera que sí reflexionan y tienen el deseo deliberado de hacer algo, pero no actúan de acuerdo a lo que han elegido porque se encuentran bajo la influencia de un deseo contrario que su perspectiva racional no aprueba. En efecto, 
en el razonamiento del incontinente son las pasiones las que determinan los fines, los cuales no encajan en la visión del sujeto del bien ni con su plan de vida en general (Burnyeat 1980, 83). Pero, ¿por qué sus acciones no concuerdan con sus premisas?, ¿acaso actúan voluntariamente? Ocurre que los incontinentes sufren un conflicto interno entre la parte racional y la apetitiva del alma en la que el deseo vence a la razón. El estagirita sugiere que probablemente esto sucede porque no han internalizado el conocimiento, de modo que hablan como si fueran actores en un teatro (Aristóteles 1985, 1147a24). Si bien algunos tachan las acciones que se realizan por ira o apetito de involuntarias e irracionales, para Aristóteles son voluntarias, pero admite que "no es fácil transformar con la razón un hábito antiguo profundamente arraigado en el carácter" (1985, 1179b16). Sin embargo, antes había asegurado que "en el caso del justo y del licencioso, podían, en un principio, no llegar a serlo y, por eso, lo son voluntariamente; pero, una vez que han llegado a serlo, ya no les es posible no serlo" (Aristóteles 1985, 1114a20-22). Respecto a su responsabilidad moral deducimos de esta afirmación que aquellos que se dejan llevar por sus pasiones, a pesar de no tener control sobre sí mismos, son efectivamente responsables porque ese es el modo de vida al que ellos mismos se acostumbraron a vivir. En un principio ellos decidieron seguir esa dirección y ya no pueden volver atrás: por eso son responsables ahora también. Probablemente, la única manera de ser compadecido y quizás eximido de parte de la culpa es si se muestra arrepentimiento, porque demuestra que sí puede ser curable. A diferencia de la acción viciosa, la akrasía puede entenderse como un tipo de inmadurez moral. El incontinente sabe lo que es mejor para él y lo que debe hacer, pero aun así no lo hace porque no tiene autocontrol. En cambio, al vicioso no le importa hacer el bien, se ha formado en el hábito de elegir mal y sin culpa. El vicioso sería en todo caso un incontinente en sentido absoluto, es decir, no de una cosa en particular, sino en todo sentido.

Aristóteles da a entender que cree en la posibilidad de modificar algunas disposiciones del carácter debido a las continuas referencias a los premios y castigos. No obstante, no solo es necesario que el sujeto relacione el dolor que le produce el castigo con las acciones malas, sino que muestre un arrepentimiento sincero. Les da mucha importancia a los estados emocionales, como ya hemos visto, porque estos revelan las intenciones. Así 
pues, el reformar la conducta requiere tiempo. Evidentemente, así como aprendemos un hábito realizándolo, lo moldeamos del mismo modo. Esto implica también eliminar las pasiones negativas y aprender a sentir placer con las pasiones positivas. El hacer lo correcto no termina con seguir las normas morales, es también interiorizarlo y actuar guiados por nuestra propia racionalidad práctica. En palabras de Guariglia:

La virtud es la capacidad de dominio, emanada de la parte racional pero desarrollada por medio del ejercicio hasta lograr su plena posesión que nos permite refrenar nuestros deseos y temores, nuestras emociones y sensaciones internas, a fin de adecuar nuestra conducta a un canon de comportamiento impuesto y transmitido por tradición (Guariglia 1997, 196).

Tal y como lo hace notar Guariglia, nuestras pasiones no dependen de nosotros, pero nuestro comportamiento frente a ellas sí, por lo que "se nos elogia o censura no por nuestras pasiones (...), sino por nuestras virtudes y vicios" (Aristóteles 1985, 1106a 1-5). En otras palabras, somos responsables de nuestros hábitos de elegir, ya sea si nos dejamos llevar o si controlamos las pasiones. Asimismo, a partir de las deliberaciones que se repiten en varias situaciones, se van formando costumbres y tradiciones éticas. En este sentido, Aristóteles no trata de establecer leyes absolutas. Lo más cercano que nos proporciona a una guía de cómo debemos actuar es el modelo del hombre prudente. Él no actúa de acuerdo a una teoría ni a un universal, basa sus juicios en su experiencia de las condiciones del ser humano y siempre tiene en cuenta el contexto particular (Nussbaum 1995, 374-375). Por eso, la ética es una sabiduría práctica y no teórica. Es la experiencia la que nos permite reconocer ciertos rasgos que se repiten y podemos generalizar para facilitar la puesta en práctica de una vida buena. Por ejemplo, nos damos cuenta cuando una situación requiere valor o cuando otra es injusta (Nussbaum 1995, 382), pero de todos modos es fundamental ser flexible y deliberar respetando la singularidad de cada caso y nuestro propio término medio de las distintas acciones.

El papel de las leyes morales es, desde la perspectiva de Aristóteles, regular los hábitos de los jóvenes cuando la educación que reciben de sus padres es insuficiente. Así pues, deben ser la encarnación de las virtudes y 
buenas costumbres. Entonces, podríamos considerar a las leyes como un instrumento para igualar las condiciones de los ciudadanos, independientemente de la educación que hayan recibido, con el fin de formar en todos hábitos virtuosos. Esto se podría prestar para una interpretación relativista, pues si el éthos es la base de una legislación, cada comunidad tendría su ética y política propias. Sin embargo, Aristóteles es en principio universalista porque a pesar de este sesgo culturalista defiende que todos los seres humanos tenemos la misma naturaleza: "cada uno debería haber nacido con un poder, como lo es el de la visión, para juzgar rectamente y elegir el bien verdadero" $(1985,1114 b 5)$. La idea de un bien verdadero suena contradictorio después de afirmar tantas veces que la ética se enfoca en las cosas particulares y contingentes. Pero la ética aristotélica, a pesar de ser universalista, no está determinada totalmente. Las normas morales sirven de guía, de modo que uno no tenga que estar deliberando profundamente antes de tomar cada decisión de su vida. Con un hábito ya establecido con la práctica, Aristóteles no aboga por una formación condicionada de la conducta establecida a través de la repetición irreflexiva, esta debe "ser complementada con una educación filosófico-teorética que dé al discípulo una comprensión del «por qué» así como del «qué»" (Nussbaum 1999, 589).

De este modo, gracias a las leyes se podría justificar el juzgar a todos los ciudadanos de la misma manera porque significa que tienen una base común. Además, esto permite resolver en parte el problema de las diferentes condiciones de educación durante la infancia. Así, si alguien fue criado de manera incorrecta, durante su madurez moral puede guiarse por las leyes para aspirar a la virtud y reformar su carácter, por lo que no se libraría de la responsabilidad moral sobre sus acciones.

\section{§IV. Conclusiones}

En conclusión, a pesar de no postular el concepto de responsabilidad moral, Aristóteles desarrolló una detallada teoría de la misma a partir de un examen de la acción intencional. Así, hemos argumentado que si combinamos su teoría de la voluntariedad con la de la elección deliberada y su análisis acerca de las disposiciones del carácter, encontramos un 
pensamiento moral vigente hasta el día de hoy. Debido a la diversidad de los tipos de acciones, clasificarlas resulta un procedimiento complejo, ya que no pueden ser todas evaluadas de la misma manera. Por lo general, el agente es moralmente responsable si el arché de la acción se encuentra en él mismo, si conoce las circunstancias en las que se produce la acción y si tiene la capacidad de elegir. Esto último es fundamental porque evidencia que una acción impulsiva también puede ser juzgada moralmente. Con respecto a las acciones involuntarias, son perdonables cuando la ignorancia es la causa, pero si la causa es una pasión que no es ni natural ni humana, no son perdonables. Sería interesante profundizar nuestro análisis enfocándonos además en las personas con trastornos psicológicos. Queda aquí abierta la cuestión concerniente, por ejemplo, a los psicópatas. Ellos actúan voluntariamente y por elección, conociendo las circunstancias de la acción. Sin embargo, ¿podemos decir que son responsables? ¿Está en su poder ser psicópatas o no?

Asimismo, Aristóteles hace unos aportes notables en cuanto al carácter. A diferencia de sus contemporáneos que creían que los dioses les infundían sus estados mentales, él define la virtud moral como un hábito electivo. De este modo, les devuelve la responsabilidad moral a los humanos por sus creencias, pasiones y modos de ser. Así pues, si bien estamos determinados a actuar según nuestro carácter, podemos considerarnos responsables porque en cierta medida, a pesar de la influencia de múltiples factores externos e internos, tenemos la capacidad de cambiar, de reformarnos y de educarnos para controlar nuestros impulsos. El estagirita resalta en este sentido la racionalidad crítica que nos permite moldear nuestros deseos e ir formándonos el hábito del buen vivir. En efecto, mientras que los animales solo se guían por placer o dolor, los humanos poseemos la capacidad de actuar en base a juicios y elegir a partir de deseos deliberados. Esto nos otorga un poder sobre nosotros mismos y sobre nuestro destino. 


\section{Bibliografía}

Aristóteles, 1985. Ética Nicomáquea, Ética Eudemia. Traducción de Julio Pallí Bonet. Madrid: Gredos.

Baracchi, Claudia, 2008. Aristotle's Ethics as First Philosophy. Nueva York: Cambridge University Press.

Bravo, Francisco, 2004. Teoría aristotélica de la responsabilidad. Revista Episteme NS, $24(2)$.

Burnyeat, M. F, 1980. Aristotle on Learning ro Be Good. En: Essays on Aristotle's Ethics, ed. Amelie Rorty. Berkley: University of California Press, 69-92.

Esponda, Katherine, 2016. Sobre la responsabilidad en la ética aristotélica. Praxis Filosófica (43), 129-154.

Guariglia, Osvaldo, 1997. La ética en Aristóteles o la moral de la virtud. Buenos Aires: Editorial Universitaria de Buenos Aires.

Irwin, Terence H, 1980. Reason and Responsibility in Aristotle. En: Essays on Aristotle's Ethics, ed. Amelie Rorty. Berkeley: University of California Press, 117-155.

Nussbaum, Martha, 1995. Aristóteles: la fragilidad de la vida buena del ser humano. En: La fragilidad del bien. Fortuna y ética en la tragedia y la filosofía griega. Madrid: La balsa de la Medusa, 310-372.

- 1999. La ética de la virtud: una categoría equívoca. Areté, XI (1-2), 573-613.

Wiggins, David, 1980. Deliberation and Practical Reason. En: Essays on Aristotle's Ethics, ed. Amelie Rorty. Berkley: University of California Press, 221-240. 